В. С. Гевод, д.х.н., профессор кафедры технологии неорганических веществ и экологии, e-mail: aquilegya@ua.fm

И. А. Борисов, студент,

А. С. Чернова, студентка

Украинский государственный химико-технологический университет пр. Гагарина, 8, г. Днепр, 49005, Украина

\title{
АДСОРБЦИЯ ПОВЕРХНОСТНО-АКТИВНЫХ ВЕЩЕСТВ НА ВОЗДУШНЫХ ПУЗЫРЬКАХ И ВЛИЯНИЕ СООТНОШЕНИЯ ИХ РАЗМЕРОВ НА ПРОИЗВОДИТЕЛЬНОСТЬ ПУЗЫРЬКОВО-ПЛЕНОЧНОЙ ЭКСТРАКЦИИ
}

Особенности пузырьково-пленочной экстракции рассмотрены с позиций динамики и равновесия адсорбции поверхностно-активных веществ (ПАВ) на пузырьках воздуха с разными размерами. Показано, что при определенном соотночении количества и размеров пузырьков, адсорбирующих и переносящих ПАВ из объема очищаемой воды в пузырьково-пленочный экстрактор, производительность процесса пузырьково-пленочной экстракции многократно возpacmaem.

Ключевые слова: вода, очистка, адсорбичя, пузырьково-пленочная экстракиия.

Введение. При решении нарастающих проблем урбанизированных территорий и, в том числе, проблем, связанных со вторичным загрязнением питьевой воды в сетях централизованного водоснабжения, всё большее значение приобретают знания, практическая реализация которых наносит минимальный ущерб окружающей среде.

В рамках экологического императива разрабатываются новые технологии и совершенствуются известные.

Одним из экологичных способов улучшения качества воды на периферии протяженных водопроводов, а также при доочистке промышленных и бытовых сточных вод и подготовке воды в домохозяйствах является использование устройств, принцип действия которых основан на реализации сопряженных процессов флотации (пузырьково-пленочной экстракции) и биологической фильтрации. Биофильтр и пузырьково-пленочный экстрактор, объединенные в эмерджентный водоочистительный комплекс, эффективно очищают воду без применения расходуемых материалов и этим обеспечивают существенную экономию материальных ресурсов на протяжении своего жизненного цикла.

1. Равновесие и динамика адсорбции ПАВ на воздушных пузырьках. Термодинамика адсорбции ПАВ в водных системах разработана Гиббсом, который раскрыл взаимосвязь поверхностных избытков ПАВ с их объ- емной концентрацией и поверхностным натяжением раствора. Мономолекулярную адсорбцию различных поверхностно-активных веществ на твердых и жидких поверхностях исследовал Ленгмюр [1]. Его уравнения изотерм адсорбции адекватно отображают сущность изучаемых явлений и широко используются в технологических расчетах.

Равновесие и кинетика адсорбции ПАВ на воздушных пузырьках рассмотрены ниже с использованием представлений Ленгмюра о том, что на любой поверхности имеется конечное количество центров (мест), потенциально доступных для адсорбции. Каждое равновесное состояние адсорбции характеризуется определенной степенью заполнения адсорбционной поверхности молекулами адсорбтива. При этом уравнение, описывающее адсорбцию в динамике, является следующим:

$$
\Gamma_{\tau}=\Gamma_{\infty}\left(\frac{C}{\frac{K \downarrow}{K \uparrow} C_{s}^{\infty}+C}\right)\left\{1-\exp \left[-(K \uparrow+K \downarrow) \frac{l}{V}\right]\right\},(1)
$$

где $\Gamma_{\tau}$ - адсорбция ПАВ на поверхности пузырьков воздуха, которая устанавливается ко времени $\tau=l / V(l-$ длина всплытия пузырьков воздуха в водной среде, $V$ - скорость всплытия пузырьков); $\Gamma_{\infty}-$ предельная адсорбция ПАВ,$C_{s}^{\infty}$ - предельная объемная концентрация ПАВ в водной среде, $C$ - исходная концентрация ПАВ в водной среде, $K \uparrow$ и $K \downarrow-$ константы скоростей адсорбции и десорбции ПАВ. 
Уравнение (1) позволяет проанализировать особенности процессов флотационного (пузырьково-пленочного) изъятия поверхностно-активных веществ из водных сред с помощью потока пузырей диспергированного воздуха или другого подходящего газа.

2. Аэрационно-индуцированный перенос ПАВ. Производительность пузырьковой флотации ПАВ зависит от: скорости адсорбции этих веществ на газовых (воздушных) пузырьках, суммарной площади адсорбционной поверхности, создаваемой всеми пузырьками в их потоке в объеме воды, скорости всплытия пузырьков под действием архимедовой силы, интенсивности гидродинамических возмущений в зоне аккумулирования флотопродукта и способа отведения флотоконцентрата за пределы флотатора (пузырьково-пленочного экстактора). Каждый из перечисленных факторов является важным.

Если поток диспергированного воздуха состоит из пузырьков примерно одинакового размера и адсорбция флотируемых примесей воды на воздушных пузырьках за время их существования в водной среде составляет величину $\Gamma_{\tau}$, то аэрационно-индуцированный перенос ПАВ из объема воды на её поверхность описывается уравнением

$$
\frac{d G}{d \tau}=\Gamma_{\tau} \frac{W_{\text {воз } 4 \pi r^{2}}}{4 \pi r^{3} / 3}=\Gamma_{\tau} \frac{3 W_{\text {возд }}}{r},
$$

где $d G$ - элементарный перенос массы ПАВ с пузырьковым потоком; $\Gamma_{\tau}$ - адсорбция ПАВ на поверхности воздушных пузырьков за время их существования в водной среде (описывается уравнением (1)); $W_{\text {возд }}$ - расход воздуха на барботаж; $r$ - радиус воздушных пузырьков; $d \tau-$ элементарное время.

Уравнения (1) и (2) показывают, что на величину $d G / d \tau$ влияют концентрация ПАВ в водном объеме $(C)$, константа адсорбционного равновесия $\left(K_{\text {равн }}=\frac{K \downarrow}{K \uparrow}\right)$, расход воздуха на барботаж $(W)$, размеры воздушных пузырьков $(r)$ и продолжительность $(l / V)$ времени существования воздушных пузырьков в объеме воды,

При заданном расходе воздуха на барботаж $\left(W_{\text {возд }}\right)$ величина произведения $\Gamma_{\tau} \frac{3 W_{\text {возд }}}{r}$ в уравнении (2) растет пропорционально уменьшению радиуса воздушных пузырьков $(r)$ и это способствует нию $d G / d \tau$. Но с уменьшением радиуса воздушных пузырьков возрастает и продолжи- тельность времени их всплытия сквозь толщу воды [2], как показано на рисунке 1. Это обеспечивает дополнительную адсорбцию мелкими пузырьками тех поверхностноактивных примесей воды, у которых коэффициенты диффузии малы. Поэтому на $d G / d \tau$ дополнительно прирастает и за счет влияния фактора $(l / V)$, входящего в экспоненциальный член выражения, представленного в уравнении (1) в фигурных скобках.

К росту $d G / d \tau$ приводит и увеличение суммы констант скоростей адсорбции и десорбции ПАВ, т. е $(K \uparrow+K \downarrow)$, входящей в (1) в показатель экспоненты.

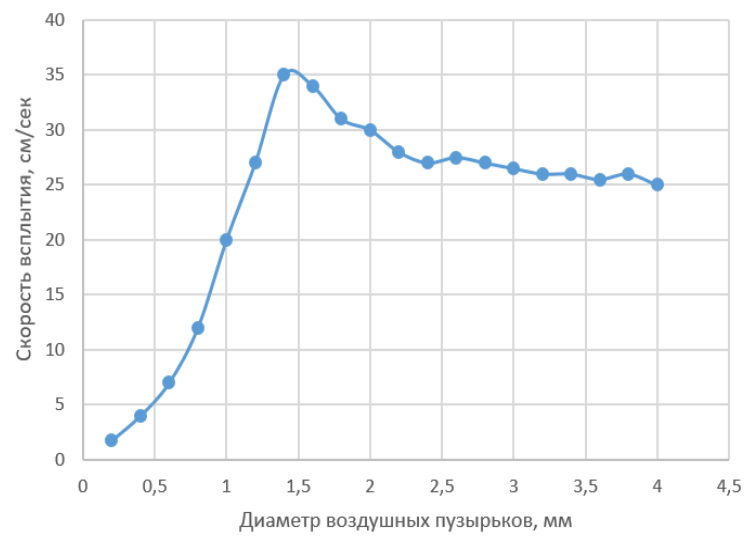

Рисунок 1 - Влияние размеров воздушных пузырьков на скорость их всплытия в воде

Но, применяя метод флотации на практике для очистки воды от её поверхностноактивных примесей, нельзя управлять параметрами $\left(\Gamma_{\infty}\right),\left(C_{S}^{\infty}\right),(K \uparrow)$ и $(K \downarrow)$ без кардинального нарушения исходного ионносолевого состава обрабатываемой воды.

Концентрация адсорбирующихся примесей $(C)$ также не является управляющим параметром.

Целенаправленно менять $d G / d \tau$ позволяют только величины $(l),(W)$ и $(r)$. Величина $(l)$ должна быть такой, чтобы достигалась максимальная степень адсорбции ПАВ пузырьками воздуха за время их всплытия в объеме воды. А что касается расхода воздуха на барботаж $(W)$ и размеров $(r)$ пузырьков, которые образуются при диспергировании воздуха, то здесь появляются ограничения, связанные со спецификой накопления и съема флотоконцентрата с поверхности субфазы. В частности, при размерах воздушных пузырьков, соответствующих скоростям их всплытия в диапазоне $25-35$ cм/c (см. рисунок 1), про- 
порционально расходу воздуха на барботаж увеличивается интенсивность гидродинамических возмущений поверхностного слоя субфазы. Это делает невозможным накопление флотоконцентрата на водной поверхности при малых концентрациях поверхностноактивных примесей в воде. А если увеличивается расход воздуха на барботаж при микроскопических размерах получаемых воздушных пузырьков, скорости всплытия которых составляют 0,1-1 cм/c, то все аэрируемое водное пространство заполняется циркулирующей водно-воздушной эмульсией. В такой эмульсии флотоконцентрат как таковой при малых концентрациях ПАВ в воде практически не образуется. При указанных условиях не образуют устойчивых слоев пены и потоки воздушных пузырьков с радиусами 0,1-1.0 мм, скорости всплытия которых варьируют в диапазоне 1-20 см/с. Эти обстоятельства ограничивают возможность применения ординарной пузырьковой флотации для очистки воды от её поверхностно-активных загрязнений.

3. Пузырьково-пленочная экстракция с использованием моно- и полидисперсных потоков воздушных пузырьков. В работах [3-7] было показано, что усовершенствованный метод пузырьковой флотации - пузырьково-пленочная экстракция, основанная на ином способе удаления флотоконцентрата с поверхности субфазы, - обеспечивает существенно более глубокую очистку воды от поверхностно-активных примесей, чем флотация с удалением флотоконцентрата в виде пенного слоя. В частности, при пузырьковой флотации образование пены прекращается, когда концентрация ПАВ в водном объеме опускается до уровня 1-2 мг/дм³. А при пузырьково-пленочной экстракции концентрацию ПАВ в воде можно понизить и до уровня микрограммов на кубический дециметр. Принцип действия пузырьково-пленочной экстракции отображен на рисунке 2, где:

a) адсорбция поверхностно-активных примесей воды потоком пузырьков воздуха и образование из этих примесей мономолекулярного слоя ПАВ на водной поверхности;

b) формирование зачатков пены. Образующиеся полусферические оболочки покрыты адсорбционными монослоями ПАВ с обеих сторон;

c) процесс внутри пузырьковопленочного экстрактора с оптимальными размерами. Из потока воздушных пузырьков с
ПАВ, адсорбированными на их поверхности, образуется поток тонких жидкостных пленок концентрата удаляемых загрязнений. Строение пленок показано на выделенном фрагменте;

$d$, e) процессы внутри цилиндрических пространств с неоптимальными размерами. В случае $(d)$ пузырьковые сферы разрушаются, не образуя воздушно-пленочного потока концентрата удаляемых загрязнений, а в случае (e) возникает тривиальное явление аэролифта.

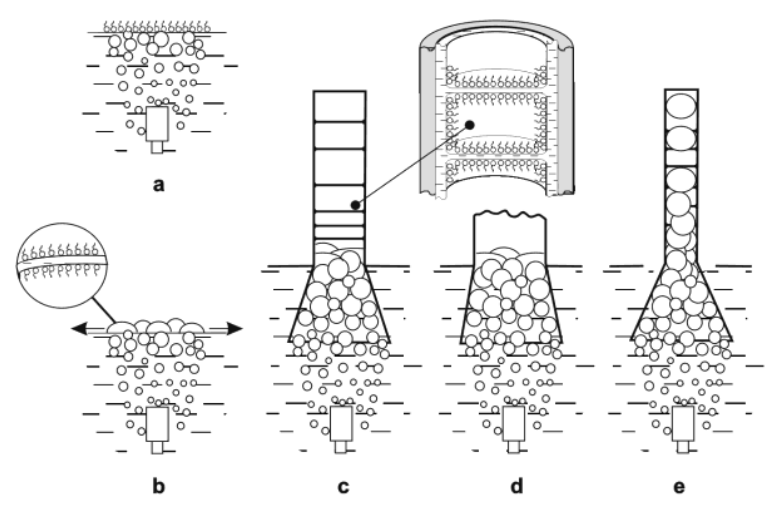

Рисунок 2 - Иллюстрация принципа действия пузырьково-пленочной экстракции

При осуществлении пузырьковопленочной экстракции концентрат сфлотированных загрязнений отводится за пределы очищаемого водного объема в виде воздушнопленочного потока через канал (патрубок) с коническим сужением на его выходе и раструбом (захватывающей воронкой) у основания (см. рисунок 2, c). В раструбе пузырьки воздуха с адсорбированными ПАВ образуют зону критического газонаполнения, коалесценцируют и, смещаясь в пространство отводящего канала, трансформируются в поток тонких жидкостных пленок. Пленки содержат в своем составе определенное количество удаляемых ПАВ и эвакуируются за пределы аэрируемого объема воды через выходное отверстие отводящего канала. Эта часть отводящего канала на рисунке 2, $c$ не показана.

Пленки разделены между собой воздушными пространствами (прослойками), как показано на выделенном фрагменте рисунка 2, c. Давление воздуха в воздушных прослойках чуть больше атмосферного и уменьшается в направлении от основания отводящего канала к его выходу. 
Объем воздушных прослоек зависит от размеров флотирующих пузырьков и от величины адсорбции ПАВ $\left(\Gamma_{\mathrm{c}}\right)$ на их поверхности к моменту вхождения в раструб. Связь жесткая, так как каждая пленка концентрата загрязнений и каждая воздушная прослойка появляются в отводящем канале в результате высвобождения определенного количества ПАВ и определенного объема воздуха из соответствующего количества скоалесцировавших воздушных пузырьков. Поскольку все пузырьки диспергируемого воздуха собираются раструбом и попадают в отводящий канал пузырьково-пленочного экстрактора, то воздушно-пленочный поток перемещается там со скоростью

$$
\bar{V}=W_{\text {возд }} / S .
$$

Здесь: $\bar{V}$ - линейная скорость перемещения жидкостных пленок по отводящему каналу; $S$ - площадь поперечного сечения отводящего канала.

В соответствии с (3) на аэрационноиндуцированный поток ПАВ расход диспергируемого воздуха должен влиять прямо пропорционально, а радиус флотирующих воздушных пузырьков - обратно пропорционально.

Исходя из этого, можно было ожидать, что увеличение расхода воздуха на барботаж и уменьшение размеров воздушных пузырьков будут обеспечивать повышение производительности пузырьково-пленочной экстракции. Но с уменьшением диаметра воздушных пузырьков до размеров менее миллиметра скорость их коалесценции даже при критическом газонаполнении кардинально уменьшается. В результате пузырьковая масса заполняет все пространство отводящего канала и движется по нему с большим количеством захваченной воды. Схематически этот процесс отображен на рисунке 2 , е. Снижение расхода воздуха на барботаж при его диспергировании в пузырьки с диаметром субмиллиметровых размеров не улучшает ситуации.

Но картина существенно меняется, когда пузырьковый поток, входящий в раструб отводящего канала, оказывается состоящим (по объему подаваемого воздуха) на $60-80 \%$ из пузырьков с диаметром 2-4 мм и на 20-40 \% из пузырьков с диаметром существенно менее 1 мм. При этом в зоне раструба образуется газожидкостная смесь, в которой пространство между крупными пузырьками (их диаметр
- 2-4 мм) заполняется мелкими пузырьками (образуется газожидкостная система вложенного типа, как схематично показано на рисунке $3, d$ ). В такой системе фактором, препятствующим коалесценции пузырьков, является электростатическое отталкивание двойных электрических слоев и электроосмотическое расклинивающее давление.
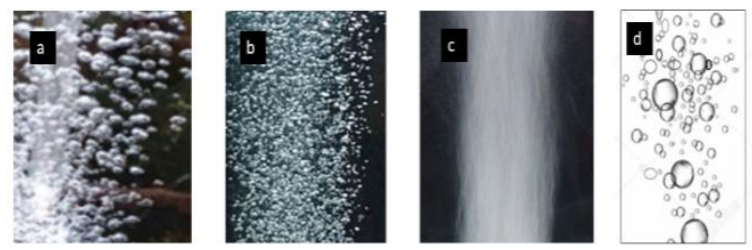

Рисунок 3 - Фотографии монодисперсных потоков воздушных пузырьков $(a, b, c)$

и иллюстрация фрагмента полидисперсного потока $(d)$, образующего вложенную структуру в захватывающей воронке пузырьковопленочного экстрактора

Двойные электрические слои всегда существуют на любых межфазных поверхностях. В пузырьковом столбе, ограниченном стенками пузырьково-пленочного экстрактора, заряды противоположных знаков сконцентрированы на пузырьковых оболочках и в водных пространствах (менисках), прилегающих к ним. При утоньшении менисков двойные электрические слои перекрывают друг друга, и между пузырьками возникает электростатическое отталкивание, которое препятствует их коалесценции. При этом происходит перераспределение ионов и электрических потенциалов внутри менисков. Увеличение концентрации ионов в менисках при перекрытии двойных электрических слоев создает локальное осмотическое давление, и вода устремляется внутрь менисков, противодействуя их утоньшению. Таким образом, к собственно кулоновским силам отталкивания добавляется осмотическая компонента расклинивающего давления.

Теория электростатического отталкивания двойных электрических слоев разработана в середине прошлого века Б. В. Дерягиным, Л. Д. Ландау, Е. Фервеем (Е. Verwey), Дж. Овербеком (D. Overbeek). Авторами показано, что при взаимодействии плоскопараллельных объектов с низкими значениями их поверхностных потенциалов $\left(\varphi_{o}\right)$ величина кулоновской составляющей расклинивающего давления определяется по уравнению

$$
\Pi_{\mathrm{e}} \approx\left(4 z^{2} e^{2} \varphi_{\mathrm{o}}^{2} n_{\mathrm{o}} / k T\right) \exp (-æ h),
$$


где $z$ - заряд противоионов, $e-$ заряд электрона, $k T-$ энергия теплового движения ионов, æ - параметр, характеризующий толщину ионной атмосферы $\delta=1 / æ, h-$ величина зазоpa между плоскостями локализации поверхностных зарядов, $n_{\mathrm{o}}-$ концентрация ионов в зазоре.

Согласно (4) расклинивающее давление $\Pi_{\mathrm{e}}$ при указанных условиях является пропорциональным квадрату поверхностного потенциала $\left(\varphi_{\mathrm{o}}\right)$.

Для сильно заряженных поверхностей, когда поверхностный потенциал $\varphi_{\mathrm{o}}>4 k T / z e$, величина электростатической компоненты расклинивающего давления не зависит от $\varphi_{о}$ и находится из следующего выражения:

$$
\Pi_{\mathrm{e}} \approx 64 n_{\mathrm{o}} k T \exp (-æ h) .
$$

При более строгом подходе к анализу сил электростатического отталкивания между двойными электрическими слоями в жидкостной пленке по теории ДЛФО получается следующая зависимость:

$$
\Pi_{\mathrm{e}} \approx 2 n_{\mathrm{o}} k T \operatorname{ch}(0,5 z e \varphi h / k T)-2 n_{0} k T .
$$

Первое слагаемое в (6) отображает осмотическое давление внутри собственно зоны слипания пузырьков, а второе - учитывает осмотическое давление в окружающем мениске. На практике раздельное определение этих двух компонент невозможно.

Специфический характер взаимодействия пузырьков проявляется и при изменении $\mathrm{pH}$. В зависимости от $\mathrm{pH}$ меняется степень ионизации многих веществ, являющихся эндогенными поверхностно-активными загрязнениями воды. В результате меняются расклинивающие давления.

Но особенностью коллективного поведения крупных и мелких пузырьков воздуха в зоне их коалесценции внутри пузырьковопленочного экстрактора является то, что крупные пузырьки, лопаясь, стимулируют процесс разрушения мелких пузырьков. Это происходит потому, что разрушение пузырьков воздуха на поверхности воды сопровождается мощными гидродинамическими и аэродинамическими возмущениями. На рисунках 4, a и 4, б показаны события, сопровождающие прекращение жизни воздушных пузырьков с радиусами примерно 5 мм и 0,5 мм, когда они лопаются на водной поверхности $[9,10]$.
Теоретические исследования этих феноменов выполнены в работах [11-14]. Установлено, что естественное разрушение пузырьков воздуха при их переходе из водной среды в окружающее воздушное пространство сопровождается генерированием капиллярных волн, и при этом исчезающий пузырек порождает быстро трансформирующийся жидкостный конус, как это отображено на фотографиях рисунка 4.

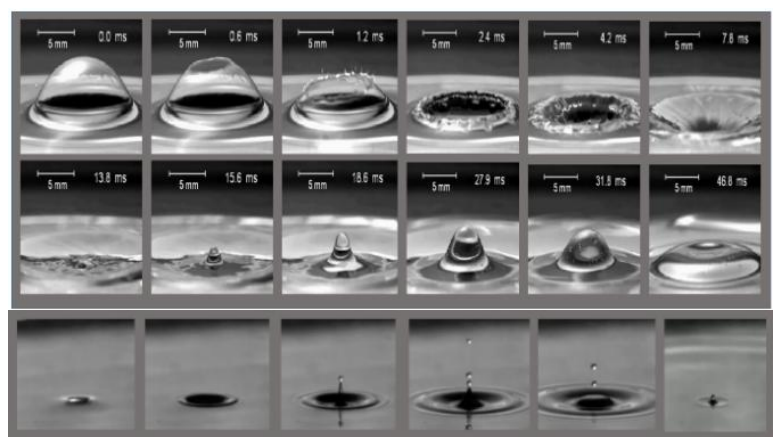

Рисунок 4 - События, сопровождающие прекращение жизни пузырьков воздуха с радиусами около 5 мм и 0,5 мм при их разрыве на поверхности воды $[9,10]$

При определенных размерах лопающихся пузырьков с вершины конуса в окружающее пространство вылетают одна или несколько микроскопических капель воды, которые в зависимости от своих размеров и начальной скорости либо возвращаются назад, либо образуют аэрозоль в воздухе. Расчетные профили изменения формы воздушного пузырька, прекращающего свое существование на поверхности воды, показаны на рисунке 5, $a$, а на рисунке 5, $b$ показана взаимосвязь между радиусами выбрасываемых микрокапель и размерами разрушающихся пузырьков.
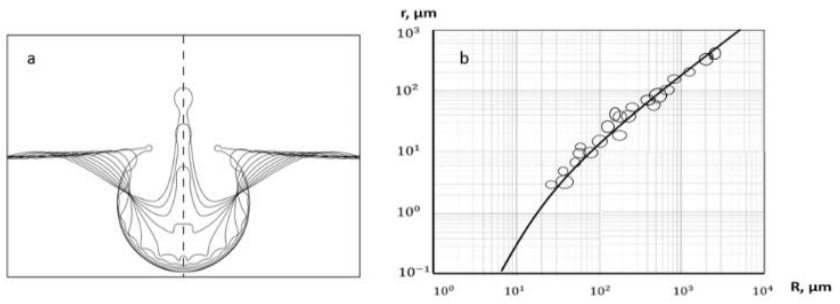

Рисунок 5 - Профили формы воздушного пузыря, который перестает существовать на поверхности воды $(a)$, и взаимосвязь между радиусами выбрасываемых капель и размерами разрушающихся воздушных пузырьков (б) 
Капиллярные волны, которые генерируются при разрушении крупных пузырьков в верхней части зоны критического газонаполнения в раструбе отводящего канала, передают импульсы силы мелким пузырькам вложенной структуры и тем самым инициируют их коалесценцию с высвобождением адсорбированных ПАВ в ту часть отводящего канала, где формируется воздушно-пленочный поток флотоконцентрата.

Уравнение, описывающее аэрационноиндуцированный поток ПАВ, который трансформируется в воздушно-пленочный поток флотоконцентрата, в этом случае имеет вид:

$$
\begin{aligned}
& \frac{d G}{d \tau}=\Gamma_{\infty}\left(\frac{C}{C_{s}^{\infty} \frac{K \downarrow}{K \uparrow}+C}\right) 3 W_{\text {возд }} * \\
& \left\{1-\exp \left[-(K \uparrow+K \downarrow) \frac{l}{\underline{\underline{V_{1}}}}\right]\right\} \frac{1-\alpha}{r_{1}}+ \\
& \left\langle\left\{1-\exp \left[-(K \uparrow+K \downarrow) \frac{l}{\underline{\underline{V_{1}}}}\right]\right\} \frac{\alpha}{r_{2}},\right.
\end{aligned}
$$

где $r_{1}$ и $r_{2}$ - радиусы воздушных пузырьков крупной и мелкой фракций, соответственно; $V_{1}$ - скорость всплытия пузырьков крупной фракции; $(1-\alpha)$ и $(\alpha)$ - доля пузырьков крупной и мелкой фракций в их потоке, а остальные символы имеют те же обозначения, что и в уравнении (1).

В уравнении (7) скорость всплытия мелких пузырьков не фигурирует, поскольку мелкие пузырьки в полидисперсном потоке приобретают примерно такую же скорость перемещения к зеркалу субфазы, что и скорость всплытия крупных пузырьков. Поэтому (7) можно представить так:

$$
\begin{gathered}
\frac{d G}{d \tau}=\Gamma_{\infty}\left(\frac{C}{C_{S}^{\infty} \frac{K \downarrow}{K \uparrow}+C}\right) 3 W_{\text {возд }} * \\
*\left\langle\left\{1-\exp \left[-(K \uparrow+K \downarrow) \frac{l}{\underline{\underline{V_{1}}}}\right]\right\}\left(\frac{1-\alpha}{r_{1}}+\frac{\alpha}{r_{2}}\right)\right\rangle .
\end{gathered}
$$

А если на пузырьках воздуха адсорбируются ПАВ с высокими коэффициентами их диффузии в воде, то (8) сводится к следующему уравнению:

$$
\begin{gathered}
\frac{d G}{d \tau}=\Gamma_{\infty}\left(\frac{C}{C_{S}^{\infty} \frac{K \downarrow}{K \uparrow}+C}\right) 3 W_{\text {возд }}\left(\frac{1-\alpha}{r_{1}}+\frac{\alpha}{r_{2}}\right)= \\
=\frac{C \cdot h}{K_{\text {равн }}} 3 W_{\text {возд }}\left(\frac{1-\alpha}{r_{1}}+\frac{\alpha}{r_{2}}\right)= \\
=K_{1} C\left(\frac{1-\alpha}{r_{1}}+\frac{\alpha}{r_{2}}\right)
\end{gathered}
$$

где $K_{1}=\frac{3 W_{\text {возд }} h}{K_{\text {равн }}}$.

Уравнение (9) показывает, как меняется доставка поверхностно-активных примесей воды в раструб отводящего канала пузырьково-пленочного экстрактора в зависимости от количества и размеров крупных и мелких пузырьков воздуха в их флотирующем потоке. В частности, если доля воздуха в крупных и мелких пузырьках составляет соответственно $70 \%$ и $30 \%$ от его общего расхода, а радиусы крупных и мелких пузырьков соотносятся как 10:1, то по сравнению со случаем, когда весь воздух расходуется на создание только крупных пузырьков, получаем увеличение $d G / d \tau$ в 3,7 раза. Это показывает расчет

$\left(\frac{1-\alpha}{r_{1}}+\frac{\alpha}{r_{2}}\right)=\frac{1}{r_{1}}\left(\frac{1-0,3}{1}+\frac{0,3}{0,1}\right)=\frac{3,7}{r_{1}}$,

где $(1-\alpha)=0,7$ - доля диспергируемого воздуха в крупных пузырьках; $\alpha=0.3$ - доля диспергируемого воздуха в мелких ках; $r_{1} / r_{2}=10 / 1$ - отношение радиуса крупных пузырьков к радиусу мелких пузырьков.

Однако при переносе ПАВ из объема воды на её поверхность концентрация ПАВ (C) в водном объеме уменьшается, и уравнение баланса имеет вид

$$
\frac{d G}{d \tau}=-\frac{V d C}{d \tau}
$$

где $V$ - объем воды, подвергаемый флотационной обработке.

Объединив уравнения (9) и (11) и сведя в этих уравнениях постоянные величины в обобщенную константу скорости процесса пузырьково-пленочной экстракции, $K_{1}=$ $\frac{3 W_{\text {возд }} h}{K_{\text {равн }}}$ для случая малых концентраций ПАВ в очищаемой воде, получим

$$
\int_{C_{0}}^{C} \frac{d C}{C}=\int_{0}^{\tau} \frac{\Gamma_{\infty}}{K_{P}} \cdot \frac{3 W_{B}}{r} \cdot \frac{d \tau}{V},
$$


где

$$
\frac{\Gamma \infty}{K_{p}} \cdot \frac{3 W_{b}}{r}=\tilde{K}
$$

И, в итоге:

$$
\frac{C}{C_{0}}=\exp \left(-\frac{\tilde{K} \tau}{V}\right)
$$

А объединив уравнения (9) и (11) с учетом (10) и выполняя те же условия и процедуры, как отмечено выше, получим

$$
\frac{C}{C_{0}}=\exp \left(-\frac{K^{*} \widetilde{K} \tau}{V}\right),
$$

где $K^{*}$ - поправочный коэффициент, который учитывает вклад мелких пузырьков в процессе пузырьково-пленочной экстракции.

Уравнение (14) раскрывает динамику пузырьково-пленочной экстракции ПАВ из заданного объема воды, когда используется поток пузырьков воздуха с существенно различающимися размерами.

На рисунке 6 представлены результаты расчетов эффективности пузырьковопленочной экстракции, отображающие динамику удаления ПАВ из водопроводной воды потоком крупных пузырьков $\left(r=2 * 10^{-3} \mathrm{M}\right)$ и потоком, состоящим из смеси крупных и мелких пузырьков $\left(r_{1}=2 * 10^{-3} \mathrm{M}, r_{2}=2 * 10^{-4} \mathrm{M}\right.$ в соотношении 7:3).

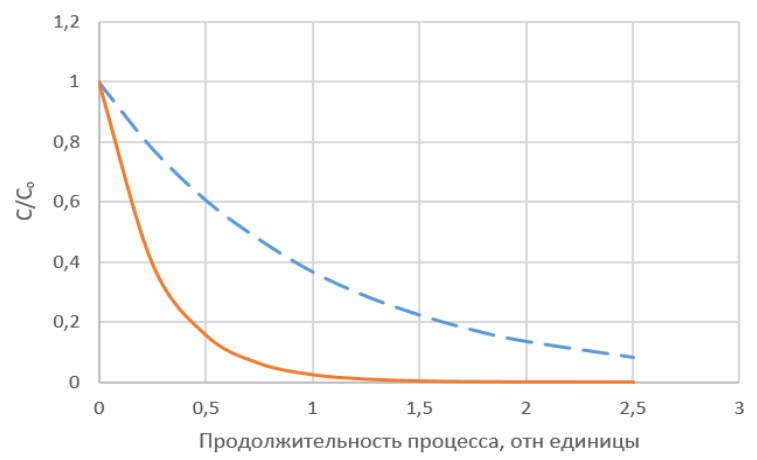

Рисунок 6 - Отношение текущих концентраций $(C)$ флотируемых поверхностно-активных примесей воды к их начальным значениям $\left(C_{0}\right)$ в зависимости от времени протекания флотационного процесса при использовании крупных пузырьков во флотационном потоке (- - - ) и смеси крупных пузырьков с мелкими пузырьками (-------) в соотношении 7:3 при одинаковых расходах воздуха на барботаж

Выводы. Пузырьково-пленочная экстракция как усовершенствованный вид барботажной флотации позволяет снизить концентрацию поверхностно-активных примесей в воде до уровня десятых долей миллиграмма на литр. Использование полидисперсного потока воздушных пузырьков при пузырьково-пленочной экстракции существенно ускоряет процесс очистки воды, когда доля крупных и мелких пузырьков воздуха достигает определенного соотношения в их смешанном потоке. Наилучший случай - когда пузырьки воздуха разных размеров образуют встроенную структуру внутри собирающей воронки отводящего канала пузырьково-пленочного экстрактора. Во встроенной структуре капиллярные волны, сопровождающие разрыв крупных пузырьков в верхней части критической зоны газонаполнения внутри воронки экстрактора пузырьковых пленок, передают свои импульсы силы пузырькам малого размера. Эти импульсы преодолевают электростатическое отталкивание в граничных менисках контактирующих пузырьков и тем самым инициируют их слияние с высвобождением большего количества адсорбированных поверхностно-активных веществ в ту часть отводящего канала в пузырьково-пленочном экстракторе, где появляется поток плоских жидких пленок флотационного концентрата. Как следствие, происходит существенное увеличение скорости очистки воды от её поверхностно-активных загрязнений.

\section{Список литературы}

[1] E. A. Moelwyn-Hughes, Physical chemistry, Pergamon Press, 1961.

[2] S. Guet, and G. Ooms, "Fluid mechanical aspects of the gas-lift technique", Annual. Rev. Fluid Mechanics, vol. 38, pp. 225-249, 2006.

[3] В.С. Гевод и др., Поверхностноактивные и другие загрязнения в водопроводной питьевой воде: свойства, мониторинг, причины накоплений и экономичное удаление, Днепропетровск: Издво УГХТУ, 2002.

[4] V.S. Gevod, Bubble-film extraction fundamentals and application: handbook of surface and colloid chemistry, third edition, ed. K. S. Birdi, CRC Press, 2009, ch. 14, pp. 585-563.

[5] V. S. Gevod, and I. L. Reshetnyak, Water purification devices: state-of-the art review, handbook of surface and colloid chemistry, fourth edition, ed. K. S. Birdi, CRC Press, 2015, ch. 8, pp. 481-542. 
[6] В. С. Гевод, и С. В. Гевод, Водоочистка по приничипу вращающегося колеса. Днепропетровск: Грани, 2015.

[7] В. С. Гевод, Опасные загрязнения водопроводной воды и их экономичное удаление. Lambert academic publishing, 2018 (ISBN 978-613-9-93414-0).

[8] I. Cantat, S. Cohen-Addad, F. Elias, F. Graner, R. Hohler, O. Pitois, et al., Foams - structure and dynamics. Oxford University Press, 2013.

[9] E. Ghabache, A. Antkowiak, C. Josserand, and T.Seon, "On the physics of fizziness: how bubble bursting controls droplets ejection", Physics of Fluids, 26, 121701, 2014. [Online]. Available: https://doi.org/10.1063/ 1.4902820 .

[10] J. Collins, Bursting bubble-slow motion. [Online]. Available: https://www. youtube.com/watch? $\mathrm{v}=$ dj10HOkkRC4

[11] C. T. Nguyen, H. M. Gonnermann, Y. Chen, C. Huber, A. A. Maiorano, A. Gouldstone, and J. Dufek, "Film drainage and the lifetime of bubbles", Geochemistry, Geophysics, Geosystems, 14 (9), pp.3616-3631, 2013.

[12] E. Ghabache, A. Antkowiak, C. Josserand, and T. Séon, "On the physics of fizziness: how bubble bursting controls droplets ejection", Physics of Fluids, 26, 121701, 2014.

[13] C. Frederik Brasz, Casey T. Bartlett, Peter L. L. Walls, Elena G. Flynn, Yingxian Estella $\mathrm{Yu}$, and James C. Bird, "Minimum size for the top jet drop from a bursting bubble", Physical Review Fluids, 3, 074001, 2018.

[14] Laurent Duchemin, Stephane Popinet, Christophe Josserand, and Stephane Zaleskia, "Jet formation in bubbles bursting at a free surface", Physics of fluids, vol. 14, no. 9, pp. 3000-3008.

\section{References}

[1] E. A. Moelwyn-Hughes, Physical chemistry, Pergamon Press, 1961.

[2] S. Guet, and G. Ooms, "Fluid mechanical aspects of the gas-lift technique", Annual. Rev. Fluid Mechanics, vol. 38, pp. 225-249, 2006.

[3] V.S. Gevod et al., Surface-active and other contaminants in tap drinking water: properties, monitoring, reasons for accumulations and economical disposal, Dnepropetrovsk: Izd-vo UGHTU, 2002 [in Russian].

[4] V.S. Gevod, Bubble-film extraction fundamentals and application: handbook of surface and colloid chemistry, third edition, ed. K. S. Birdi, CRC Press, 2009, ch. 14, pp. $585-563$.

[5] V.S. Gevod, and I. L. Reshetnyak, Water purification devices: state-of-the art review, handbook of surface and colloid chemistry, fourth edition, ed. K. S. Birdi, CRC Press, 2015, ch. 8, pp. 481-542.

[6] V.S. Gevod, and S. V. Fevod, Rotary wheel water treatment. Dnepropetrovsk: Grani, 2015 [in Russian].

[7] V.S. Gevod, Hazardous pollution of tap water and its economical disposal. Lambert academic publishing, 2018 (ISBN 978-6139-93414-0) [in Russian].

[8] I. Cantat, S. Cohen-Addad, F. Elias, F. Graner, R. Hohler, O. Pitois, et al., Foams - structure and dynamics. Oxford University Press, 2013.

[9] E. Ghabache, A. Antkowiak, C. Josserand, and T. Seon, "On the physics of fizziness: how bubble bursting controls droplets ejection", Physics of Fluids, 26, 121701, 2014. [Online]. Available: https://doi.org/10.1063/ 1.4902820 .

[10] J. Collins, Bursting bubble-slow motion. [Online]. Available: https://www. youtube.com/watch? $\mathrm{v}=\mathrm{dj} 10 \mathrm{HOkkRC} 4$

[11] C. T. Nguyen, H. M. Gonnermann, Y. Chen, C. Huber, A. A. Maiorano, A. Gouldstone, and J. Dufek, "Film drainage and the lifetime of bubbles", Geochemistry, Geophysics, Geosystems, 14 (9), pp. 3616-3631, 2013.

[12] E. Ghabache, A. Antkowiak, C. Josserand, and T. Séon, "On the physics of fizziness: how bubble bursting controls droplets ejection", Physics of Fluids, 26, 121701, 2014.

[13] C. Frederik Brasz, Casey T. Bartlett, Peter L. L. Walls, Elena G. Flynn, Yingxian Estella $\mathrm{Yu}$, and James C. Bird, "Minimum size for the top jet drop from a bursting bubble", Physical Review Fluids, 3, 074001, 2018.

[14] Laurent Duchemin, Stephane Popinet, Christophe Josserand, and Stephane Zaleskia, "Jet formation in bubbles bursting at a free surface", Physics of fluids, vol. 14, no. 9, pp. 3000-3008. 
V. S. Gevod, I. A. Borisov, A. S. Chernova

\title{
ADSORPTION OF SURFACE-ACTIVE SUBSTANCES ON AIR BUBBLES AND THE INFLUENCE OF THEIR SIZES RELATIONSHIP ON THE PERFORMANCE OF BUBBLE-FILM EXTRACTION
}

\begin{abstract}
Bubble-film extraction as an improved form of bubbling flotation allows to reduce the concentration of surface-active impurities in water to the level of tenths of a milligram per liter. The use of a polydisperse stream of air bubbles during bubble-film extraction significantly accelerates the process of water purification, when the proportion of large and small air bubbles reaches a certain ratio in their mixed stream. The best case is when air bubbles of different sizes form an integrated structure inside the collecting funnel of the discharge channel of the bubble-film extractor. In the built-in structure, capillary waves accompanying the rupture of large bubbles in the upper part of the critical gas-filling zone inside the funnel of the bubble film extractor transmit their pulses to small-sized bubbles. These pulses overcome the electrostatic repulsion in the boundary menisci of the contacting bubbles and thereby initiate their fusion with the release of more adsorbed surface-active substances into that part of the discharge channel in the bubble-film extractor where a stream of flat liquid films of flotation concentrate appears. As a result, a significant increase in the rate of water purification from its surface-active pollution occurs. The features of bubble-film extraction (enhanced bubble flotation) for water purification are considered from the standpoint of the equilibrium and dynamics of adsorption of surface-active admixtures of water (surfactants) on air bubbles with various sizes. It is shown, that within a certain ratio of dimensions and quantity of air bubbles in their stream transferring surfactants from the water bulk into the bubble-film extractor, the productivity of bubble-film extraction process increases many times. The reason consists in the collective fusion of big and small air bubbles in their embedded structure inside of the bubble-films extractor under the action of capillary waves appearing at the bursting of large bubbles.
\end{abstract}

Keywords: water, purification, adsorption, bubble-film extraction.

\author{
В. С. Гевод, І. О. Борисов, А. С. Чернова
}

\section{АДСОРБЦІЯ ПОВЕРХНЕВО-АКТИВНИХ РЕЧОВИН НА ПОВІТРЯНІ БУЛЬБАШКИ І ВПЛИВ СПІВВІДНОШЕННЯ ЇХ РОЗМІРІВ НА ПРОДУКТИВНІСТЬ БУЛЬБАШКОВО-ПЛІВКОВОЇ ЕКСТРАКЦІї}

Біофільтр і бульбашково-плівковий екстрактор, об'єднані в емерджентний водоочисний комплекс, ефективно очищають воду без застосування вартісних матеріалів, забезпечуючи суттєву економію матеріальних ресурсів протягом свого життєвого ииклу. Особливості отримання бульбашкової плівки для очищення води розглядаються у статті з точки зору рівноваги та динаміки адсорбиї̈ поверхнево-активних домішок води на бульбашках повітря різного розміру. Отримання бульбашкової плівки як вдосконаленої форми барботажної флотації дає змогу знизити концентраиію поверхнево-активних домішок у воді до рівня десятих мг на літр. Використання полідисперсного потоку повітряних бульбашок під час вилучення плівки значно прискорює прочес очищення води, коли частка великих $і$ малих бульбашок повітря досягає певного співвідношення в їх змішаному потоиі. Найкращий випадок, коли бульбашки різного розміру утворюють інтегровану структуру всередині збиральної воронки каналу зливу плівки витяжски. У вбудованій структурі капілярні хвилі, що супроводжують розрив великих бульбашок у верхній частині критичної зони наповнення газом всередині воронки витяжки плівки, передають свої імпульси бульбашкам маленьких розмірів. Ці імпульси долають електростатичне відштовхування в межових менісках контактуючих бульбашок і тим самим ініціюють їх злиття з виділенням більше адсорбованих поверхнево-активних речовин у ту частину каналу розряду в плівковій витяжсиі, де з'являсться потік плоских рідких плівок флотаційного концентрату. Внаслідок иього відбувається значне збільшення швидкості очищення води від ї̈ поверхнево-активного забруднення. Показано, щзо в певному співвідношенні розмірів $i$ кількості повітряних бульбашок у потоці, щуо переносить поверхнево-активні речовини з маси води в бульбашкову плівкову витяжску, продуктивність процесу вилучення плівки збільшується в багато разів. Причина полягає в колективному зрощенні великих і малих бульбашок повітря у їх вбудованій структурі всередині бульбашкових плівок витяжки під дією капілярних хвиль, щэо виникають при лопанні великих бульбамок.

Ключові слова: вода, очищення, адсорбиія, бульбашково-плівкова екстракиія.

Стаття надійшла 27.06.2019

Прийнято 19.07.2019

(C) В. С. Гевод, И. А. Борисов, А. С. Чернова, 2019

DOI: $10.24025 / 2306-4412.3 .2019 .178304$ 